\title{
Meteorology for Coastal/Offshore Wind Energy in the United States \\ Recommendations and Research Needs for the Next 10 Years
}

by Cristina l. Archer, Brian A. Colle, luca Delle Monache, Michael J. Dvorak, Julie lundquist,

Bruce H. Bailey, Philippe Beaucage, Matthew J. Churchfield, Anna C. Fitch, Branko Kosovic, Sang Lee, Patrick J. Moriarty, Hugo Simao, Richard J. A. M. Stevens, Dana Veron, and John Zack

0 ffshore wind energy harvesting is just starting in the United States, with imminent offshore wind farms in Massachusetts, Maryland, and Rhode Island waters, and with an ambitious goal of $10 \mathrm{GW}$ of installed offshore capacity by 2020 set by the U.S. Department of Energy (DOE), which has recently funded seven "Advanced Technology Demonstration" offshore wind projects to help achieve that goal. Although new in the United States, offshore wind energy harvesting began more than 20 years ago in Europe and has now reached more than 5.5 GW of installed capacity worldwide, predominantly in Denmark and the United Kingdom. Given the unfortunate coincidence of introducing a new industry

AFFILIATIONS: ARCHER AND VERON-University of Delaware, College of Earth, Ocean, and Environment, Newark, Delaware; Colle-Stony Brook University/SUNY, Stony Brook, New York; Delle Monache, Fitch, AND Kosovic-National Center for Atmospheric Research, Boulder, Colorado; DvoraK-Sailor's Energy, Berkeley, California; LUNDQUIST-University of Colorado at Boulder, Boulder, Colorado, and National Renewable Energy Laboratory, Golden, Colorado; BAILEY, BeAUCAGE, AND ZACKAWS Truepower, LLC, Albany, New York; ChURCHFIELD, LeE, AND MORIARTY - National Renewable Energy Laboratory, Golden, Colorado; SIMAO—Princeton University, Princeton, New Jersey; Stevens-Johns Hopkins University, Baltimore, Maryland, and University of Twente, Enschede, The Netherlands CORRESPONDING AUTHOR: Cristina L. Archer, University of Delaware, College of Earth, Ocean, and Environment, Newark, DE 19716

E-mail: carcher@udel.edu

DOI:10.1175/BAMS-D-I3-00108.I

A supplement to this article is available online (I0.II75/BAMS-D-13-00I08.2).

(C2014 American Meteorological Society

\section{during challenging economic times, it is essential that public and private financial resources be effectively and optimally directed toward those meteorologi- cal research needs that are emerging today and that will be critical in the next decade. Identifying these research needs for wind energy along the U.S. East Coast, both coastal and offshore, was the goal of a two-day symposium held at the University of Dela- ware on 27-28 February 2013. More than 40 partici- pants gathered from academia, national laboratories, wind industry, and funding agencies. \\ During the symposium, three main topics were explored: 1) wind resource assessment; 2) wind power forecasting; and 3) turbulent wake losses. Overviews of the latest findings in the three topics were given on the first day in the form of presentations, which were open to students and the general public. On the sec- ond day, the experts gathered in a workshop to iden- tify research needs and provide recommendations for urgent action items. Whereas specific research needs were identified for each of the three main topics, two emerged as crosscutting and urgent: 1) continuous, publicly available, multilevel measurements of winds and temperature over U.S. offshore waters; and 2) quantification and reduction of uncertainty. These two research needs and relevant recommendations (in italics) are described first. \\ RESEARCH NEED \#I: MORE OFFSHORE} OBSERVATIONS. Offshore meteorological measurements are challenging and expensive. Ideal measurements would quantify the wind resource at several vertical levels spanning the height of the turbine rotor disk to understand the rotor equivalent wind speed and possible impacts on turbine power 
production. In European waters, designated research platforms (e.g., FINO1 in Germany) have been established for characterization of offshore flow as well as validation of new measurement technologies such as light detection and ranging (lidar) and modeling approaches. The few long-term meteorological observations off the East Coast are typically buoy-based, thereby restricting the altitude of wind measurements to a few meters above the surface. A sparse network of nine towers, with an elevation of $\sim 50 \mathrm{~m}$, extends along the coast from Florida to Maine, but fails to provide multilevel information and measurements at turbine hub height or above.

Periodically, detailed measurements of wind and temperature have been conducted offshore in shortterm field campaigns, but the consistent long-term measurements required for resource assessment are generally not available off the East Coast (with the only exception being the Cape Wind tower in Nantucket Sound, Massachusetts). The standard approach considered buoy measurements and then extrapolated them to higher altitudes with assumptions of the shape of the wind profile (log-law or power-law). By extrapolating surface or near-surface measurements with such smooth profiles, important wind structures such as low-level jets are ignored.

The first recommendation is the deployment of a more dense network of meteorological towers, which will enable traditional resource assessment measurements such as wind speed, wind direction, and turbulence at several levels from the surface to the rotor disk top, and temperature profiles for quantifying atmospheric stratification and stability. Ideally, such towers could also provide a platform for validating remote-sensing measurements. The U.S. DOE has proposed the Reference Facility for Offshore Renewable Energy (RFORE) to be located at the Chesapeake Light Tower, approximately 13 miles off the Virginia Coast. The facility provides a first step toward addressing the shortage of offshore wind data.

Beyond meteorological towers, remote-sensing technology mounted either on fixed towers or on floating platforms could provide data over broader regions. Scanning Doppler lidar, wind-profiling lidar, and sodar can provide valuable wind speed and direction measurements throughout the turbine rotor disk and beyond. Radiometers can quantify temperature and humidity profiles to determine atmospheric stability.

In addition to long-term measurements of winds, temperature, and moisture profiles, short-term intensive measurement campaigns with a broader deploy- ment of instruments would also be of value, especially for model validation.

These recommendations for more intensive observations extend a prior call for more onshore meteorological observations and focused field campaigns made by DOE in 2008. Since then, new types of remote-sensing instruments have become more widely available and more accepted in the wind energy industry for wind resource characterization.

\section{RESEARCH NEED \#2: UNCERTAINTY CHARACTERIZATION. Deterministic wind} power forecasts based on numerical weather prediction (NWP) can provide useful information for decision making. However, by design, a single plausible future state of the atmosphere starting from a single initial state is generated. Imperfect initial and boundary conditions and model deficiencies inevitably lead to nonlinear error growth during model integration. Accurate knowledge of the continuum of plausible future states [the forecast probability density function (PDF)] is considerably more useful for decision making because it allows for a quantification of the uncertainty associated with a forecast.

"Ensembles" are used today to generate a set of plausible future atmospheric states and to estimate the forecast PDF of atmospheric variables relevant to wind power. Ensembles are created from the outputs of NWP models using any of the following: various initial conditions, different parameterizations within a single model, stochastic approaches with diverse numerical schemes, different models, and coupled ocean-atmosphere schemes. For wind energy, one important additional source of uncertainty comes from the challenging step of wind-to-power conversion.

Ensembles are affected by biases in the ensemble mean and by lack of diversity among the ensemble members, particularly in the planetary boundary layer (PBL). Therefore, postprocessing is an important component of the wind forecasting process and should be explored further, preferably including methods and techniques developed by the wind industry. Since the wind industry benefits from the findings published by the research community and the public sector, it is recommended that a regular two-way exchange of know-how between academia, the public sector, and industry be established to help advance the science and prevent duplication of efforts. A promising postprocessing technique is the analog approach, in which past observations that correspond to past predictions that best match selected features of the 
current forecast, such as time series of wind speed and direction, are used to correct the current forecast. Other promising techniques are advanced model output statistics (e.g., neural networks, support vector machines, and random forests).

Recently, operational centers have generated multiyear reforecast datasets to support successful calibration of both deterministic and probabilistic forecasts. It is expected that in the next few years new calibration techniques, possibly combining statistical and dynamical approaches, will lead to large improvements in the accuracy of wind power predictions and in the reliable characterization of their uncertainty.

Next, the three main topics and their associated specific research needs are described.

TOPIC\# I : RESOURCE ASSESSMENT. Initial maps of the U.S. offshore wind resource from the National Renewable Energy Laboratory (NREL) and others by Stanford University have identified gross characteristics of the hub-height offshore wind resource, which have been generally useful to policymakers and researchers and for early-stage project development. Using mesoscale modeling techniques, these maps provide estimates of wind speed and direction, diurnal and seasonal patterns, wind shear, and air density at horizontal grid scales of approximately $1-5 \mathrm{~km}$. This information, although essentially unverified due to the lack of hub-height measurements described in Research Need \#1, has enabled numerous project siting studies, wind farm layout and energy production simulations, and estimates of development potential as a factor of water depth, distance from shore, wind resource, and other factors. However, there is a need to accurately capture dynamic coastal processes, such as sea breezes, low-level jets, and other land-air-ocean interactions, as they represent a significant source of variability in the available wind.

Data representing assessment periods of 20-25 years (i.e., project lifetimes) are typically required for bankable offshore projects; interannual speed variability of $4 \%-6 \%$ is not uncommon. The probability and magnitude of extreme events-particularly peak winds and waves and hurricanes-and the effects of more common events-such as winter storms, icing from sea spray, and salt corrosion-need to be better known to properly design turbines and foundations and meet industry standards. In a changing climate, more studies are needed to reduce the uncertainty of a changing wind resource as ocean, offshore, and coastal temperatures change. Changes in the local wind environment over time may also be caused by the increasing presence of other wind farms within a given region, as described in Topic \#3.

Recent studies have explored strategic temporal, climatological, and spatial aspects of the offshore resource, including large-scale wind farm interconnection scenarios. U.S. East Coast offshore wind has been found to be particularly coincident with peak-electricity demand. Similar studies should be performed to identify resource attributes that can add value to generally higher offshore costs and evaluate the sensitivity of project location, including distance from the shore, to load coincidence.

Significant offshore resource assessment uncertainties exist. Most of the aforementioned studies relied on mesoscale modeling that was validated with generally sparse in situ data. Perhaps the largest uncertainty is extrapolating surface observations-generally $5-\mathrm{m}$ buoy anemometer measurements to heights across the turbine rotor. As such, there is an urgent need for multilevel wind and temperature observations at platforms offshore (as in Research Need \#1), equipped with either meteorological towers that are as tall or taller than hub height, or lidars. In the coastal region, transport processes (advection of either maritime air inland or continental air offshore) during sea- and land-breeze events often cause the PBL to deviate from classic well-mixed, neutrally stable conditions. Existing PBL parameterizations struggle to perform well in these conditions. Research effort is needed to improve such $P B L$ parameterizations in coastal regions.

Long-term wind climatologies require publicly available historic reanalysis data and future climate data generated by models forced under different anthropogenic emission scenarios. Most of the existing publicly available data are at a relatively coarse spatial scale $(>20 \mathrm{~km})$ compared to the size of a typical wind farm. Dynamical downscaling methods typically employ a regional climate model to generate higher spatiotemporal wind climatologies but at a high computational expense for long climate records. Stochastic downscaling methods are computationally cheaper and have been shown to accurately downscale low-resolution reanalysis data with acceptable accuracy, as compared to in situ validation data.

TOPIC \#2: WIND POWER FORECASTING. Wind power forecasting is challenging because the relationship between wind speed and power production for a single wind turbine or a wind farm is nonlinear; 
for some wind speed ranges, the sensitivity of power production forecasts to wind speed forecast error is quite high. For example, a modest $1.5 \mathrm{~m} \mathrm{~s}^{-1}$ error in a wind speed forecast can, in some cases, result in a power production forecast error of over $20 \%$ of a wind farm's capacity.

A diverse set of prediction tools and input data has been applied to the wind power forecast problem for a range of time scales. Intrahour forecasts (0$60 \mathrm{~min}$ ahead) are needed for regulation and realtime dispatch decisions. At this scale, the effects of small eddies and turbulent mixing are important but cannot be resolved by operational models. Therefore, mainly statistical methods are used, which are based on near-real-time observations. This has driven the deployment of meteorological sensors and lidars for intrahour forecasting.

The forecast 1-6 $\mathrm{h}$ ahead for load-following and next-operating-hour commitment has to account for various mesoscale weather phenomena (e.g., sea breezes, convective systems, and local topography). The rapid-update NWP approach most likely offers the best potential for improvement in this time frame. This is a tool with increasing capability, largely because of improvements in data assimilation techniques (e.g., the hybrid ensemble Kalman filter approach), the formulations of physics-based submodels, and the amount and quality of data available for assimilation. The state of the art in rapid-update systems is the High-Resolution Rapid Refresh (HRRR) model, currently undergoing experimental operation at NOAA, which assimilates the latest data and generates a $15-\mathrm{h}$ forecast on a $3-\mathrm{km}$ grid every hour.

The day-ahead forecast is important for unit commitment, scheduling, and market trading, which require knowledge of the evolving synoptic storm systems using NWP models and ensembles. The seasonal predictions for resource planning and contingency analysis require knowledge of global teleconnections (such as El Niño). These predictions are based largely on the analysis of cyclical patterns and climate forecast system models.

It is also recommended that more offshore observations be collected using towers, lidars, and buoys, to better validate models, help with data assimilation and uncertainty characterization, and improve the model physics, because many of the PBL schemes were originally developed over land. These efforts will require a close collaboration between operational forecast centers, industry, and academia.
Finally, future efforts should focus on improving the models' ability to represent the $P B L$ and the interactions of finescale processes with larger scale flows, both inland and offshore. Such improvements will be possible only with investments that focus on improving our understanding of these key processes using real observations. Several workshops over the past 20 years have noted the need for improved PBL modeling, but no concerted effort at making such improvements has been made.

\section{TOPIC \#3: TURBULENT WAKE LOSSES.}

Wind turbines generate wakes downstream, which are generally characterized by a wind speed deficit and higher turbulence than the upwind environment. Because wakes can reduce power production and increase structural fatigue in downstream turbines, understanding wake properties, quantifying resulting power losses, and optimizing wind turbine layouts to minimize such losses is especially important to the wind energy industry. Accurately modeling turbine wakes is also important for other atmospheric applications that span a wide range of spatial scales, such as the impacts of wind energy deployment on the global climate, local meteorology, crop production, and the wind resource itself.

Because atmospheric flows are characterized by high Reynolds numbers $\left(\sim 10^{7}-10^{8}\right)$, the number of grid points required to explicitly resolve such flows with operating wind turbines via direct numerical simulation is $\sim 10^{18}$, which is prohibitive in the foreseeable future. As such, the wind industry has traditionally relied on computationally efficient wake models to simulate wind turbine wakes. In order of increasing complexities, these earlier wake models include: analytical representations of the wake deficit (e.g., the PARK model); parabolized forms of the ReynoldsAveraged Navier-Stokes (RANS) equations (e.g., the Ainslie model, also called the eddy viscosity model; UPMPARK, which uses a k-E turbulence closure); hybrid models based on an internal boundary layer growth parameterization and coupled with a parabolized RANS or an analytical model (e.g., Deep-Array Wake Model and Large Array Wind Farm model); and nonlinear RANS models (e.g., WindModeller, Ellipsys, and FUGA). Although these models are attractive for their quick runtime, they have limited ability to capture the detailed wake characteristics because they are not suitable for simulations of unsteady, anisotropic turbulent flows. 
To overcome these limitations, the research community has been using large-eddy simulation (LES), in which large-scale flow structures are resolved while the effects of smaller eddies are represented with a subgrid model (Smagorinsky or dynamical). In addition, the wind turbine is represented by either an actuator disk (with or without rotation features) or by actuator lines (one per blade) that exert a force on the flow and act as a momentum sink, or by the vortex method. Arrays of multiple wind turbines, in which multiple wakes interact with one another, have also been successfully simulated with LES. However, because of the high CPU-hours required, LES can be conducted for only a few hours or at equilibrium-state using periodic boundary conditions.

Because LES models for turbine wakes were traditionally developed in-house by research centers or universities without any funds for distributing, maintaining, or testing the codes, they are generally not available to the public. The only exception is the open-source Simulator for Offshore/Onshore Wind Farm Applications (SOWFA) from NREL, which includes a finite-volume scheme, actuator disks/lines, and options for periodic or nonperiodic boundary conditions. Although developing numerous in-house LES codes is of value because researchers can obtain independent verification of results, it is recommended that more effort and funds be devoted to maintaining LES codes for turbine wakes and making them available to the public.

To avoid the steep computational costs of simulating real wind farms with high numbers of turbines via LES, parameterizations of the effects of large wind farms on regional meteorology and global climate have been developed for mesoscale NWP and large-scale climate models, which are less computationally demanding. These parameterizations represent wind farms as either an elevated momentum sink [often with an added source of turbulent kinetic energy (TKE)], increased surface roughness, or an increased surface drag coefficient. Because surface-based parameterizations incorrectly extract momentum near the surface, as opposed to around hub height, they are not recommended for turbine wake impact studies. Although the global-scale impacts of even high penetrations of wind energy have proven to be negligible, local wakes extending tens of kilometers downwind of individual large wind farms have been generated by some wind farm parameterizations. However, to date, few observations are available to verify these model results.

Comparing model results with wind tunnel experiments, with either a single turbine or multiple turbines, is useful because the constant and controllable environment in a wind tunnel can be reproduced well. However, wind tunnel conditions are different from real atmospheric conditions and therefore field measurements are also recommended both at individual turbines and at offshore wind farms. Short-term field campaigns, as well as routine measurements (especially offshore) are needed to validate results under a large umbrella of atmospheric conditions. It is recommended that inflow, near-wake, and far-wake vertical wind profiles and atmospheric stability be measured, as well as wake properties, such as TKE and turbulent fluxes (preferably with scanning lidars or arrays of sonic anemometers).

An extensive list of references for all the topics described above is available online as supplemental material (http://dx.doi.org/I0.II75/BAMS-D-I3-00I08.2).

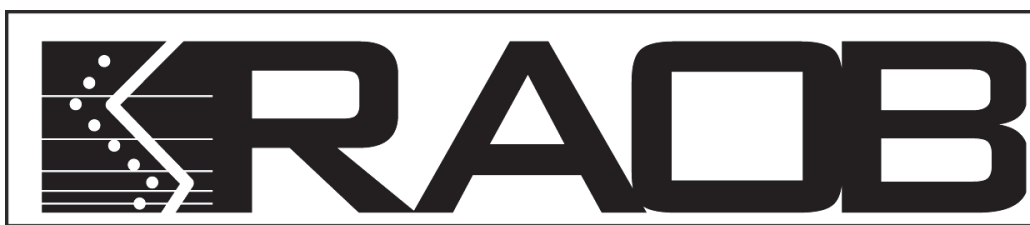

THE UNIVERSAL SOUNDING DECODE \& ANALYSIS PROGRAM

\section{Don't compromise}

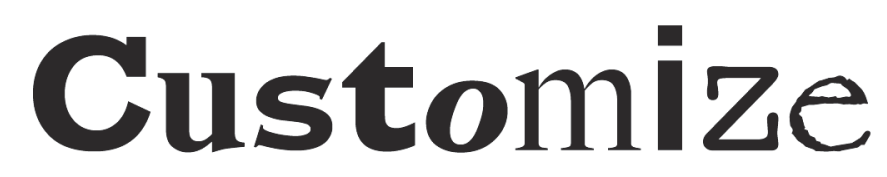

with 17 optional modules
RAOB...for the most versatile and accurate sounding analyses available! Environmental Research Services, LLC • 570-491-4689 • www.raob.com 


\section{Find out from the authoritative source}

[ What's a dust devil? ]

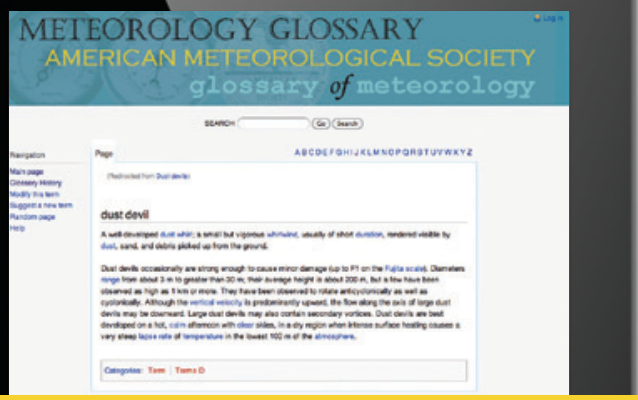

\section{Online Glossary of Meteorology}

With over 12,000 meteorological terms, you'll be able to look up definitions online any time, any place, anywhere.

http://glossary.ametsoc.org/wiki

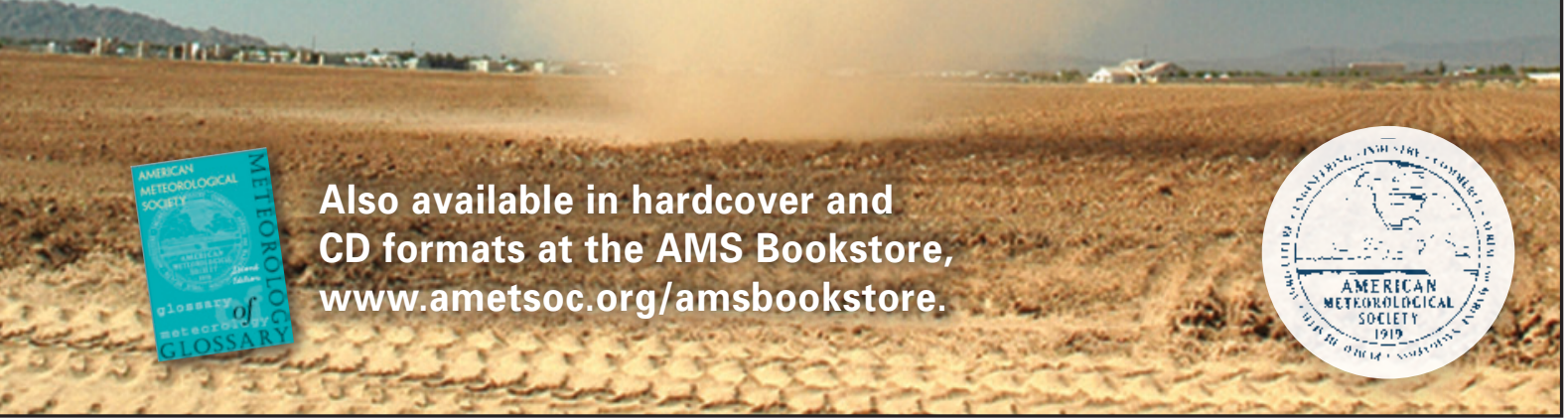

